\title{
THE ITERATED TOTAL SQUARING OPERATION
}

\author{
LUCIANO LOMONACO
}

(Communicated by Frederick Cohen)

\begin{abstract}
In this paper we prove a formula that expresses the iterated total squaring operation in terms of modular invariant theory and provide an alternative proof of a classical result of Múi's.
\end{abstract}

\section{INTRODUCTION}

We start by recalling some notation from invariant theory (see [5]). Let

$$
\begin{gathered}
P_{m}=\mathbb{F}_{2}\left[t_{1}, \ldots, t_{m}\right] ; \\
e_{m}=\prod\left(\sum_{i=1}^{m} \lambda_{i} t_{i}\right) \quad\left(\lambda_{i}=0,1, \sum \lambda_{i}>0\right) .
\end{gathered}
$$

We set $\Phi_{m}=P_{m}\left[e_{m}^{-1}\right]$. The natural action of $\mathrm{GL}_{m}\left(\mathbb{F}_{2}\right)$ on the $\mathbb{F}_{2}$-vector space spanned by $t_{1}, \ldots, t_{m}$ extends to an action on $P_{m}$ and $\Phi_{m}$ ( $e_{m}$ is fixed in this action). Let $T_{m} \leq \mathrm{GL}_{m}$ be the upper triangular subgroup. We want to consider the rings of invariants

$$
\Delta_{m}=\Phi_{m}^{T_{m}} ; \quad \Gamma_{m}=\Phi_{m}^{\mathrm{GL}_{m}} .
$$

$\Delta_{m}$ and $\Gamma_{m}$ can be described as follows. We set

$$
V_{k+1}=\prod\left(\sum_{i=1}^{k} \lambda_{i} t_{i}+t_{k+1}\right), \quad \lambda_{i}=0,1 ;
$$

$$
v_{k+1}=\frac{V_{k+1}}{e_{k}}
$$

and define the elements $Q_{m, j} \in P_{m}$ inductively with the formulae

$$
Q_{m, j}=Q_{m-1, j} Q_{m-1,0} v_{m}+Q_{m-1, j-1}^{2}
$$

subject to the conventions

$$
Q_{m, j}= \begin{cases}1 & \text { if } m=j \geq 0, \\ 0 & \text { if } j<0 \text { or } m<j .\end{cases}
$$

Received by the editors January 27, 1991.

1991 Mathematics Subject Classification. Primary 55P99.

Key words and phrases. Invariant theory, cohomology operations. 
If $I=\left(i_{1}, \ldots, i_{m}\right)$ is a multi-index, with $i_{j} \in \mathbb{Z}$, we set

$$
-I=\left(-i_{1}, \ldots,-i_{m}\right)
$$

and write $v^{I}$ for the monomial $v_{1}^{i_{1}} \ldots v_{m}^{i_{m}}$ (and similarly we write $S q^{I}$ or $S q^{\left(i_{1}, \ldots, i_{m}\right)}$ for the monomial $S q^{i_{1}} \ldots S q^{i_{m}}$, where the $S q^{j}$ 's are the Steenrod squares). In particular, we have

$$
\Delta_{m}=\mathbb{F}_{2}\left[v_{1}^{ \pm 1}, \ldots, v_{m}^{ \pm 1}\right] ; \quad \Gamma_{m}=\mathbb{F}_{2}\left[Q_{m, 0}^{ \pm 1}, Q_{m, 1}, \ldots, Q_{m, m-1}\right] .
$$

We observe that

$$
P_{m} \cong H^{*}\left(R P^{\infty} \times \cdots \times R P^{\infty}\right) \quad(m \text {-copies }) .
$$

Here and in the sequel $H^{*}$ indicates the mod 2 reduced cohomology functor. Hence $P_{m}$ is acted upon by the mod 2 Steenrod algebra $\mathscr{A}$ and such an action extends, in a unique way, to an action on $\Phi_{m}$ (see [7]). $\Phi_{m}$ is a graded object: the grading is obtained by assigning degree 1 to each of the variables $t_{1}, \ldots, t_{m}$. Now we consider the iterated total squaring operation $S_{m}$, defined as

$$
\begin{aligned}
S_{m}: H^{*}(X) & \longrightarrow \Phi_{m} \otimes H^{*}(X) \quad(X \text { a } C W \text {-complex }) \\
x & \longmapsto \sum_{i_{j} \geq 0}\left(t_{1}^{-i_{1}} S q^{i_{1}}\right) \cdots\left(t_{m}^{-i_{m}} S q^{i_{m}}\right)(x) .
\end{aligned}
$$

$S_{m}$ can be constructed in a purely algebraic way (as in [3]) or geometrically (e.g., see [2]).

Remark 1.4. If $X$ is a $C W$-spectrum, $S_{m}$ can still be defined, but $\Phi_{m} \otimes H^{*}(X)$ should be regarded as a completed tensor product (as in [1, p. 441]). In fact, when $X$ is a spectrum, $H^{*}(X)$ is a stable $\mathscr{A}$-module and $S_{m}(x)$ is, in general, an infinite sum.

In this paper we exhibit an explicit nice formula for $S_{m}(x)$ as an element of $\Delta_{m} \otimes H^{*}(X)$. We show that

$$
S_{m}(x)=\sum_{I} v^{-I} \otimes S q^{I}(x), \quad I=\left(i_{1}, \ldots, i_{m}\right) ; \quad i_{j} \geq 0 .
$$

Moreover we construct a sequence of maps

$$
\omega_{m}: \mathscr{A}_{*} \longrightarrow \Delta_{m}, \quad m \geq 1,
$$

where $\mathscr{A}_{*}$ denotes the $\mathbb{F}_{2}$-dual of $\mathscr{A}$. This construction allows us to give an alternative proof of a normalized version of a result of Múi's [3, Theorem 1, p. 346]. In fact, we show that

$$
S_{m}(x)=\sum_{R} \omega_{m}\left(\xi^{R}\right) \otimes \xi_{*}^{R}(x)
$$

where the sum runs over the multi-indices $R=\left(r_{1}, \ldots, r_{k}\right)$ such that $r_{i} \geq 0$ for each $i=1, \ldots, k$ and $k \leq m, \xi^{R}=\xi_{1}^{r_{1}} \ldots \xi_{k}^{r_{k}}$ is a monomial in $\mathscr{A}_{*}$ and $\xi_{*}^{R}$ indicates the corresponding element in the Milnor basis $\mathscr{B}$ of $\mathscr{A}$. We then show that the coefficient $\omega_{m}\left(\xi^{R}\right)$ that appears in the RHS of (1.5) equals the monomial $Q_{m, 0}^{-r_{1} \cdots-r_{k}} Q_{m, 1}^{r_{1}} \cdots Q_{m, k}^{r_{k}}$ and (1.5) becomes

$$
S_{m}(x)=\sum_{R} Q_{m, 0}^{-r_{1} \cdots-r_{k}} Q_{m, 1}^{r_{1}} \ldots Q_{m, k}^{r_{k}} \otimes \xi_{*}^{R}(x) .
$$


This is the announced normalized version of Múi's theorem. In particular, the above formula expresses the properties of invariance of the operation $S_{m}$. For related results, see also [4].

\section{A NICE FORMULA FOR $S_{m}(x)$}

This section is devoted to the proof of the following proposition.

Proposition 2.1. Let $x \in H^{*}(X)$. We have

$$
S_{m}(x)=\sum_{I} v^{-I} \otimes S q^{I}(x), \quad I=\left(i_{1}, \ldots, i_{m}\right) ; \quad i_{j} \geq 0 .
$$

Proof. As $v_{1}=t_{1}$, the statement is trivial for $m=1$. We use induction on $m$. We will assume the statement true for $m<n \quad(n \geq 2)$ and prove it for $m=n$. We have

$$
S_{n}(x)=\left(\sum_{i_{1} \geq 0} t_{1}^{-i_{1}} S q^{i_{1}}\right)\left(\sum_{i_{2}, \ldots, i_{n} \geq 0}\left(t_{2}^{-i_{2}} S q^{i_{2}}\right) \ldots\left(t_{n}^{-i_{n}} S q^{i_{n}}\right)(x)\right) .
$$

Our inductive hypothesis tells us that

$$
\begin{aligned}
S_{n-1}(x) & =\sum_{i_{j} \geq 0}\left(t_{1}^{-i_{2}} S q^{i_{2}}\right) \ldots\left(t_{n}^{-i_{n}} S q^{i_{n}}\right)(x) \\
& =\sum_{i_{j} \geq 0} v_{1}^{-i_{2}} \ldots v_{n-1}^{-i_{n}} \otimes S q^{\left(i_{2}, \ldots, i_{n}\right)}(x) \\
& =\sum_{i_{j} \geq 0} \prod_{k=1}^{n-1}\left(\frac{\prod_{\lambda_{j}=0,1}\left(\sum_{j=1}^{k-1} \lambda_{j} t_{j}+t_{k}\right)}{\prod_{\mu_{1}, \ldots, \mu_{k-1}=0,1} \sum_{j=1}^{k-1} \mu_{j} t_{j}}\right)^{-i_{k+1}} \otimes S q^{\left(i_{2}, \ldots, i_{n}\right)}(x) .
\end{aligned}
$$

In the last step above we have simply substituted each $v_{h}$ with its rational expression in the $t_{j}$ 's, using (1.1), (1.2), and (1.3). Therefore, using (2.3) and (2.4), we get

$$
S_{n}(x)=S_{1}\left(\sum_{\substack{i_{h}>0 \\ 2 \leq h \leq n}} \prod_{\substack{k=1 \\ n=1}}^{n-1}\left(\frac{\prod_{\lambda_{j}=0,1}\left(\sum_{j=2}^{k} \lambda_{j} t_{j}+t_{k+1}\right)}{\prod_{\substack{\mu_{j}=0,1 \\ \sum \mu_{i}>0}}^{k} \sum_{j=2}^{k} \mu_{j} t_{j}}\right)^{-i_{k+1}} \otimes S q^{\left(i_{2}, \ldots, i_{n}\right)}(x)\right) .
$$

In the above formula we have applied our inductive hypothesis using the set of variables $\left\{t_{2}, \ldots, t_{n}\right\}$ instead of $\left\{t_{1}, \ldots, t_{n-1}\right\}$. Since $S_{1}$ is a ring homomorphism (as is well known and easy to prove using the Cartan formula) 
we get

$$
\begin{aligned}
S_{n}(x) & =\sum_{i_{2}, \ldots, i_{n} \geq 0} \prod_{k=1}^{n-1}\left(\frac{\prod\left(\sum \lambda_{j} S_{1}\left(t_{j}\right)+S_{1}\left(t_{k+1}\right)\right)}{\prod \sum \mu_{j} S_{1}\left(t_{j}\right)}\right)^{-i_{k+1}} \otimes S_{1}\left(S q^{\left(i_{2}, \ldots, i_{n}\right)}(x)\right) \\
& =\sum_{i_{2}, \ldots, i_{n} \geq 0} \prod_{k=1}^{n-1}\left(\frac{\prod\left(\sum \lambda_{j} S_{1}\left(t_{j}\right)+S_{1}\left(t_{k+1}\right)\right)}{\prod \sum \mu_{j} S_{1}\left(t_{j}\right)}\right)^{-i_{k+1}} \otimes \sum_{i_{1} \geq 0} t_{1}^{-i_{1}} S q^{\left(i_{1}, \ldots, i_{n}\right)}(x) \\
& =\sum_{i_{1}, \ldots, i_{n} \geq 0} v_{1}^{-i_{1}} \prod_{k=1}^{n-1}\left(\frac{\prod\left(\sum \lambda_{j} S_{1}\left(t_{j}\right)+S_{1}\left(t_{k+1}\right)\right)}{\prod \sum \mu_{j} S_{1}\left(t_{j}\right)}\right)^{-i_{k+1}} \otimes S q^{I}(x) .
\end{aligned}
$$

Here the $\lambda_{h}$ 's and the $\mu_{l}$ 's are as in $(2.5), I$ stands for $\left(i_{1}, \ldots, i_{n}\right)$ and we use again the fact that $v_{1}=t_{1}$. Hence we only need to check that

$$
v_{k+1}=\frac{\prod\left(\sum \lambda_{j} S_{1}\left(t_{j}\right)+S_{1}\left(t_{k+1}\right)\right)}{\prod \sum \mu_{j} S_{1}\left(t_{j}\right)}
$$

As $t_{j}$ is a one-dimensional class, we have

$$
S_{1}\left(t_{j}\right)=t_{j}+t_{1}^{-1} t_{j}^{2}, \quad j=2, \ldots, n \quad(\text { see }[6, \text { Lemma } 2.7, \text { p. 6] })
$$

Thus

$$
\begin{aligned}
\prod_{\lambda_{j}=0,1}\left(\sum_{j=2}^{k} \lambda_{j} S_{1}\left(t_{j}\right)+S_{1}\left(t_{k+1}\right)\right) & =\prod\left(\sum \lambda_{j}\left(t_{j}+t_{1}^{-1} t_{j}^{2}\right)+t_{k+1}+t_{1}^{-1} t_{k+1}^{2}\right) \\
& =t_{1}^{-2^{k}} \cdot \prod\left(\sum \lambda_{j}\left(t_{1} t_{j}+t_{j}^{2}\right)+t_{1} t_{k+1}+t_{k+1}^{2}\right) .
\end{aligned}
$$

Similarly

$$
\prod_{\mu_{2}, \ldots, \mu_{k}=0,1} \sum_{j=2}^{k} \mu_{j} S_{1}\left(t_{j}\right)=t^{-2^{k}+1} \cdot \prod \sum \mu_{j}\left(t_{1} t_{j}+t_{j}^{2}\right)
$$

Therefore

$$
\frac{\prod\left(\sum \lambda_{j} S_{1}\left(t_{j}\right)+S_{1}\left(t_{k+1}\right)\right)}{\prod \sum \mu_{j} S_{1}\left(t_{j}\right)}=\frac{\prod\left(\sum \lambda_{j}\left(t_{1} t_{j}+t_{j}^{2}\right)+t_{1} t_{k+1}+t_{k+1}^{2}\right)}{t_{1} \cdot \prod \sum \mu_{j}\left(t_{1} t_{j}+t_{j}^{2}\right)}
$$

If we write $A$ ( $B$ respectively) for the numerator (the denominator respectively) of the RHS of (2.6) above, we want to check that

$$
A=V_{k+1} ; \quad B=e_{k}
$$


We have

$$
\begin{aligned}
V_{k+1} & =\prod_{\lambda_{j}=0,1}\left(\lambda_{1} t_{1}+\cdots+\lambda_{k} t_{k}+t_{k+1}\right) \\
& =\prod_{\lambda_{j}=0,1}\left(t_{1}+\lambda_{2} t_{2}+\cdots+\lambda_{k} t_{k}+t_{k+1}\right) \cdot \prod_{\lambda_{j}=0,1}\left(\lambda_{2} t_{2}+\cdots+\lambda_{k} t_{k}+t_{k+1}\right) \\
& =\prod_{\lambda_{j}=0,1}\left(\left(t_{1}+\lambda_{2} t_{2}+\cdots+\lambda_{k} t_{k}+t_{k+1}\right)\left(\lambda_{2} t_{2}+\cdots+\lambda_{k} t_{k}+t_{k+1}\right)\right) \\
& =\prod_{\lambda_{j}=0,1}\left(\lambda_{2} t_{1} t_{2}+\cdots+\lambda_{k} t_{1} t_{k}+t_{1} t_{k+1}+\left(\lambda_{2} t_{2}+\cdots+\lambda_{k} t_{k}+t_{k+1}\right)^{2}\right) \\
& =\prod_{\lambda_{j}=0,1}\left(\lambda_{2} t_{1} t_{2}+\cdots+\lambda_{k} t_{1} t_{k}+t_{1} t_{k+1}+\lambda_{2} t_{2}^{2}+\cdots+\lambda_{k} t_{k}^{2}+t_{k+1}^{2}\right) \\
& =\prod_{\lambda_{j}=0,1}\left(\lambda_{2}\left(t_{1} t_{2}+t_{2}^{2}\right)+\cdots+\lambda_{k}\left(t_{1} t_{k}+t_{k}^{2}\right)+t_{1} t_{k+1}+t_{k+1}^{2}\right)=A .
\end{aligned}
$$

A similar argument shows that $B=e_{k}$.

\section{AN Alternative PROOF OF A RESUlt of Múi's}

We recall that the dual of the mod 2 Steenrod algebra $\mathscr{A}$ is a graded polynomial algebra

$$
\mathscr{A}_{*}=\mathbb{F}_{2}\left[\xi_{1}, \xi_{2}, \xi_{3}, \ldots\right]
$$

with grading given by setting $\operatorname{deg}\left(\xi_{i}\right)=2^{i}-1$.

As usual, for each multi-index $R=\left(r_{1}, \ldots, r_{k}\right)$ with each $r_{i} \geq 0$, we will write $\xi^{R}$ for the monomial $\xi_{1}^{r_{1}} \ldots \xi_{k}^{r_{k}}$. As it is well known, the elements of $\mathscr{A}$ dual to the monomials $\xi^{R}$ with respect to the basis of admissible monomials form a basis $\mathscr{B}$, called the Milnor basis of $\mathscr{A}$. The element of $\mathscr{B}$ dual to $\xi^{R}$ is indicated by $\xi_{*}^{R}$.

We can define a map, which is formally identical to the iterated total squaring operation,

$$
\begin{aligned}
S_{m}: \mathscr{A} & \longrightarrow \Delta_{m} \otimes \mathscr{A} \subseteq \Phi_{m} \otimes \mathscr{A}, \\
\alpha & \longmapsto \sum_{I} v^{-I} \otimes S q^{I} \circ \alpha,
\end{aligned}
$$

with the proviso that $\Delta_{m} \otimes \mathscr{A}$ and $\Phi_{m} \otimes \mathscr{A}$ should be thought of as completed tensor products (as in Remark 1.4), because $\mathscr{A}$ is stable as a graded $\mathscr{A}$-module and $S_{m}(\alpha)$ is, in general, an infinite sum.

Definition 3.1. Let $\omega_{m}: \mathscr{A}_{*} \longrightarrow \Delta_{m}$ be defined as follows. Let $\xi \in \mathscr{A}_{*}$, i.e., $\xi: \mathscr{A} \rightarrow \mathbb{F}_{2}$ is an $\mathscr{A}$-map, where $\mathbb{F}_{2}$ has the trivial $\mathscr{A}$-action. We set

$$
\omega_{m}(\xi)=\left((\mathrm{id} \otimes \xi) \circ S_{m}\right)(1) \quad(1 \in \mathscr{A}) .
$$

In other words, $\omega_{m}$ is defined by the following diagram

$$
\begin{gathered}
\mathscr{A} \stackrel{S_{m}}{\longrightarrow} \Delta_{m} \otimes \mathscr{A} \stackrel{\text { id } \otimes \xi}{\longrightarrow} \Delta_{m} \otimes \mathbb{F}_{2} \cong \Delta_{m} \\
1 \longmapsto \omega_{m}(\xi) .
\end{gathered}
$$


As

$$
S_{m}(1)=\sum_{I} v^{-I} \otimes S q^{I} \quad(\text { an infinite sum })
$$

we have

$$
\omega_{m}(\xi)=(\mathrm{id} \otimes \xi)\left(\sum_{I} v^{-I} \otimes S q^{I}\right)=\sum_{I} v^{-I} \cdot\left\langle\xi, S q^{I}\right\rangle
$$

where $\left\langle\xi, S q^{I}\right\rangle$ is the value of the map $\xi$ on $S q^{I}$.

Proposition 3.2. $\omega_{m}$ is a ring homomorphism.

Proof. This is a straightforward calculation.

Proposition 3.3.

$$
\omega_{m}\left(\xi_{k}\right)=\sum_{I} v^{-I}
$$

where the sum runs over the multi-indices $I$ of the form

$$
I=\left(0, \ldots, 0,2^{k-1}, 0, \ldots, 0,2^{k-2}, \ldots, 1,0, \ldots, 0\right),
$$

that is, I is the multi-index $\left(2^{k-1}, 2^{k-2}, \ldots, 2,1\right)$ with $m-k$ zeros inserted somewhere.

Proof. $\quad \xi_{k}$ is dual to $M_{k}=S q^{2^{k-1}} S q^{2^{k-2}} \ldots S q^{1}$ and it is easy to check that $M_{k}$ does not appear in the admissible expression of any other monomial in $\mathscr{A}$. Therefore $\left\langle\xi_{k}, S q^{I}\right\rangle=1$ if and only if $S q^{I}=M_{k}$, i.e., if and only if $I$ is of the form (3.4).

\section{Proposition 3.5.}

$$
\omega_{m}\left(\xi_{k}\right)=Q_{m, 0}^{-1} Q_{m, k} \in \Gamma_{m} \subseteq \Delta_{m} \quad \forall m \geq 1 .
$$

Proof. See [2, Proposition 1, p. 39].

In other words, $Q_{m, 0}^{-1} Q_{m, k}$ is the sum of all monomials $v^{-I}$ with $I$ of the form (3.4). From Propositions 3.2, 3.3, and 3.5 we deduce the following statement.

\section{Corollary 3.6.}

$$
\omega_{m}\left(\xi^{R}\right)=Q_{m, 0}^{-r_{1} \ldots-r_{k}} Q_{m, 1}^{r_{1}} \ldots Q_{m, k}^{r_{k}} \quad\left(R=\left(r_{1}, \ldots, r_{k}\right)\right) .
$$

In [3] Múi defines a non-normalized version of $S_{m}$, which he calls $F_{m}$. By non-normalized we mean that $F_{m}$ does not preserve the degrees; in fact, if $x \in H^{n}(X)$, the degree of $F_{m}(x)$ is $2^{m} \cdot n$ while $S_{m}(x)$ has degree $n$. Múi proves the following result [3, p. 346].

\section{Theorem 3.7.}

$$
\begin{aligned}
F_{m}(x)=\sum_{R} Q_{m, 0}^{n-r_{1} \ldots-r_{k}} Q_{m, 1}^{r_{1}} \ldots Q_{m, k}^{r_{k}} \otimes \xi_{*}^{R}(x) & \\
& \left(R=\left(r_{1}, \ldots, r_{k}\right), r_{i} \geq 0, x \in H^{n}(X)\right) .
\end{aligned}
$$

Corollary 3.6 allows us to give a very simple alternative proof of a normalized version of Theorem 3.7 above, using $S_{m}$ instead of $F_{m}$. 


\section{Theorem 3.8.}

$$
\begin{aligned}
S_{m}(x)=\sum_{R} Q_{m, 0}^{-r_{1} \ldots-r_{k}} Q_{m, 1}^{r_{1}} \ldots Q_{m, k}^{r_{k}} \otimes \xi_{*}^{R}(x) & \\
& \left(R=\left(r_{1}, \ldots, r_{k}\right), r_{i} \geq 0, k \leq m\right) .
\end{aligned}
$$

Proof. We know, from (2.2), that

$$
S_{m}(x)=\sum_{I} v^{-I} \otimes S q^{I}(x) .
$$

But the Milnor elements $\xi_{*}^{R}$ form a basis for $\mathscr{A}$, therefore we have an expression of the form

$$
S_{m}(x)=\sum_{R} \alpha\left(\xi^{R}\right) \otimes \xi_{*}^{R}(x)
$$

where the $\alpha\left(\xi^{R}\right)$ 's are suitable elements of $\Gamma_{m} \subset \Delta_{m}$. More precisely, for each $R, \alpha\left(\xi^{R}\right)$ is the sum of all the monomials $v^{-I}$ with $I$ such that $\xi_{R}\left(S q^{I}\right)=1$, i.e.,

$$
\begin{aligned}
\alpha\left(\xi^{R}\right) & =\omega_{m}\left(\xi^{R}\right) \\
& =Q_{m, 0}^{-r_{1} \ldots-r_{k}} Q_{m, 1}^{r_{1}} \ldots Q_{m, k}^{r_{k}} \quad(\text { by Corollary 3.6) } .
\end{aligned}
$$

\section{ACKNOWLEDGMENT}

Most of the results presented in this paper have been obtained while I was a $\mathrm{Ph}$. D. student at the University of Warwick and are extracted from my thesis (see [2]). I would like to express my gratitude to my supervisor J. D. S. Jones for introducing me to the subject and for his help and guidance during my stay in Warwick.

\section{REFERENCES}

1. J. F. Adams, J. H. Gunawardena, and H. Miller, The Segal conjecture for elementary abelian p-groups, Topology 24 (1985), 435-460.

2. L. Lomonaco, Invariant theory and the total squaring operation, $\mathrm{Ph}$. $\mathrm{D}$. Thesis, University of Warwick, UK, 1986.

3. H. Múi, Dickson invariants and the Milnor basis of the Steenrod algebra, Topology and applications, Colloq. Math. Soc. János Bolyai 41 (1983), 345-355.

4. I. Madsen and R. J. Milgram, The classifying spaces for surgery and cobordism of manifolds, Ann. of Math. Stud., no. 92, Princeton Univ. Press, Princeton, NJ, 1979.

5. W. Singer, Invariant theory and the Lambda algebra, Trans. Amer. Math. Soc. 280 (1981), 673-693.

6. N. E. Steenrod and D. B. A. Epstein, Cohomology operations, Ann. of Math. Stud., no. 50 Princeton Univ. Press, Princeton, NJ, 1962.

7. C. Wilkerson, Classifying spaces, Steenrod operations and algebraic closure, Topology 16 (1977), 227-237. 